\title{
Jumping Control for Compliantly Actuated Multilegged Robots
}

\author{
Dominic Lakatos, Gianluca Garofalo, Alexander Dietrich, and Alin Albu-Schäffer
}

\begin{abstract}
A feedback control to generate jumping motions for compliantly actuated multilegged robots is proposed. The method allows to specify the direction of the jumping motion. This is achieved by a constraint that defines a one-dimensional submanifold and a bang-bang control which generates a limit cycle on this submanifold. The approach is based on classical impedance control with the difference that the stiffness on the submanifold and the force to preserve a predefined nominal body configuration result from the intrinsic mechanical springs in the joints. Furthermore, we propose two controller implementations: the first implementation does not require to detect the contact state, while the second implementation requires contact state detection, but accounts in addition for Coulomb friction constraints. The controller is validated in simulation with a compliantly actuated quadruped.
\end{abstract}

\section{INTRODUCTION}

Compliant actuators in robotic arms have been shown to robustly handle mechanical impacts and to improve the performance and energy efficiency [1], [2], [3], [4], [5], [6], [7]. Especially in the case of cyclic motion tasks, the capability to buffer and release elastic energy may reduce the size and weight of required actuators and save a substantial amount of energy. These properties are even of majorincreased importance for multilegged robots, which need to wirelessly walk, jump, or run over rough and uneven terrain. The step from rigid towards elastic actuation introduces natural oscillation dynamics into the plant which can be exploited on the one side, but it turns the control into a challenging task on the other side.

The idea of legged robots with mechanical springs in the joints has been initiated by passive dynamic walkers [8] and evolved to compliantly actuated walking, hopping, and running robots [9], [10], [11]. Thereby, the common design and control goal is to exploit the natural dynamics of the plant such that the resulting system approaches the performance, efficiency, and versatility of the biological archetypes. This paper focuses on the control of compliantly actuated legged robotic systems. Our work is inspired by experimental observations of biologists [12] who hypothesize that high-dimensional, nonlinear system dynamics anchored in a complex animal collapse to simple template dynamics like the spring loaded inverted pendulum (SLIP). These assumptions are further supported by biological evidence and robotic implementations of central pattern generation (CPG) [13] and adaptive frequency oscillators (AFO) [14]. These approaches are based on the assumption that a central unit, e. g. composed of multiple, phase coupled oscillators, is

The authors are with the Institute of Robotics and Mechatronics, German Aerospace Center (DLR), D-82234 Oberpfaffenhofen, Germany. Contact: dominic.lakatosedlr.de

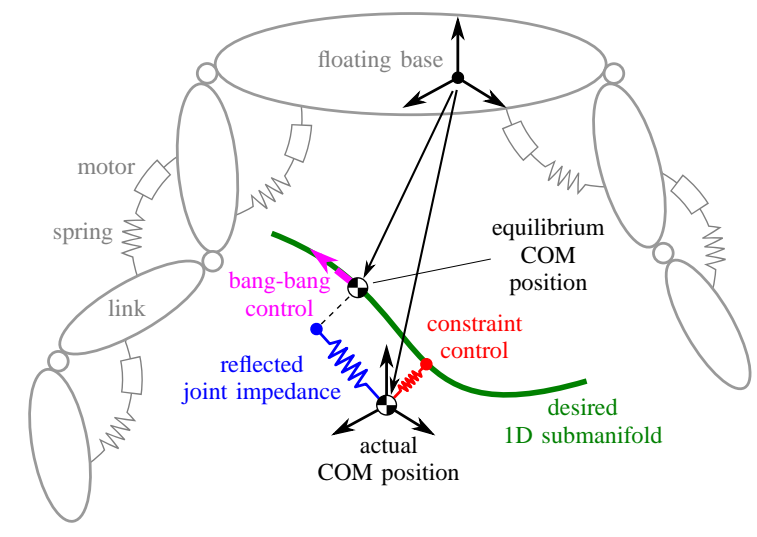

Fig. 1. General idea of the control strategy

able to generate the complex multi degree-of-freedom (DoF) motion. While in [13] the CPGs are applied as open loop control, the approach of [14] considers already a feedback of the plant in the motion pattern generation.

In our recent papers [15], [16] we proposed a control approach which directly excites the intrinsic mechanical oscillation modes of the plant. Using a switching control triggered by the torque/deflection of the springs in the joints of the robot, the frequency of the oscillation adapts to the mechanical frequency of the system. In contrast to [13], [14], in [15], [16] the oscillatory plant dynamics itself are used as oscillation unit. The resulting motion corresponds to the initially excited oscillation mode which is determined by the mechanical structure of the system. Therefore, the resulting motion is potentially energy efficient.

In this paper we present a control method which makes use of the beneficial properties of the switching control, but additionally allows to predefine the shape of the oscillation mode by control. This is achieved by specifying a onedimensional submanifold of the Cartesian space. One of the main contributions is that the controller is designed such that the elastic behavior of the springs in the joints is changed only to a minimum extent by control, i. e. only to approach the desired submanifold. As exemplified in the simulation part of the paper, the user can control the direction of the jumping motion. This is the main difference of the current method compared to our previous work [15], [16], where the oscillation mode is completely determined by the structure of the plant.

The paper is structured as follows: In the next section we present the general idea of the method. Then, in Sect. III we briefly introduce the considered model which is then used in Sect. IV as a basis for the controller design. In Sect. V 
the method is validated by simulation and finally Sect. VI concludes the work.

\section{THE IDEA}

The control strategy introduced here aims at generating predefined jumping motions by exploiting the intrinsic mechanical properties of the plant. The class of systems considered in this work are floating base systems with a number of compliantly actuated limbs, where the tip motion of the limbs is subject to contact constraints such that the floating base system can be statically balanced ${ }^{1}$. As sketched in Fig. 1, each joint of the limbs is equipped with a motor which acts on the succeeding link via a spring.

The basic idea is controlling the amplitude and direction of the net force acting on the center of mass (COM) via the position of the motors in the joints such that a desired jumping motion results. As depicted in Fig. 1, our controller is based on a Cartesian impedance at the COM that reflects the mechanical impedance of the springs in the joints as close as possible. More precisely, the impedance acts between the actual COM position which reflects the configuration of the limbs and the equilibrium COM position which reflects the configuration of the motors. If the actual and the equilibrium $\mathrm{COM}$ position are not equal, the impedance produces a Cartesian force at the COM. Thereby, the direction of the force depends on the direction of the corresponding control error. In order to control the direction of the Cartesian force, the motion of the Cartesian control error can be constrained to a predefined, one-dimensional submanifold. Therefore, this submanifold determines the direction of the Cartesian force acting at the COM. As in [17], we push the system to a one-dimensional submanifold (defined by the control law), where we generate a limit cycle. However, here the oscillation is not generated based on energy considerations, but using the bang-bang control introduced in [15], [16], we are able to adapt to the mechanical oscillation frequency of the system.

\section{MODEL}

Consider the legged floating base system with a kinematic structure as shown in Fig. 2. The position and orientation of the base link frame $\{\mathcal{B}\}$ with respect to a world coordinate system $\{\mathcal{W}\}$ is described by $\boldsymbol{r}_{\mathrm{b}} \in \mathbb{R}^{3}$ and $\boldsymbol{R}_{\mathrm{b}} \in S O(3)$, respectively, and the configuration of the legs is given by the joint coordinates $\boldsymbol{q} \in \mathcal{Q} \subset \mathbb{R}^{n}$ with $3<n \in \mathbb{N}$. The generalized velocity of the complete system $\boldsymbol{v}=\left(\boldsymbol{\omega}^{T} \boldsymbol{\nu}^{T} \dot{\boldsymbol{q}}^{T}\right)^{T}$ is composed of the angular velocity $\boldsymbol{\omega} \in \mathbb{R}^{3}$ and translational velocity $\boldsymbol{\nu} \in \mathbb{R}^{3}$ of the floating base, and the joint velocity $\dot{\boldsymbol{q}} \in \mathbb{R}^{n}$ for all actuated joints. In the following, we consider

\footnotetext{
${ }^{1}$ Note that at least three contact points are required (which are not all aligned) to statically balance a spatial free floating base system against gravity.
}

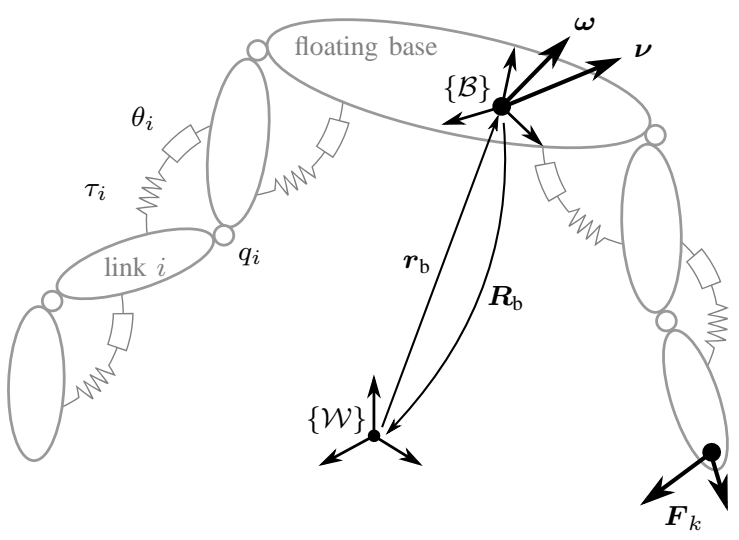

Fig. 2. Kinematic structure of the legged floating base system

generic dynamic systems satisfying

$$
\begin{array}{r}
\boldsymbol{M}\left(\boldsymbol{q}, \boldsymbol{R}_{b}\right) \dot{\boldsymbol{v}}+\boldsymbol{p}\left(\boldsymbol{q}, \boldsymbol{v}, \boldsymbol{R}_{b}\right) \\
=\left(\begin{array}{c}
\mathbf{0} \\
\mathbf{0} \\
\boldsymbol{\tau}-\boldsymbol{d}(\dot{\boldsymbol{q}})
\end{array}\right)+\sum_{k} \boldsymbol{J}_{k}(\boldsymbol{q})^{T} \boldsymbol{F}_{k},
\end{array}
$$

where $\boldsymbol{M}\left(\boldsymbol{q}, \boldsymbol{R}_{b}\right) \in \mathbb{R}^{(6+n) \times(6+n)}$ represents the inertia matrix, $\boldsymbol{p}\left(\boldsymbol{q}, \boldsymbol{v}, \boldsymbol{R}_{b}\right) \in \mathbb{R}^{6+n}$ represents the vector of Coriolis, centrifugal and gravity forces, and $\boldsymbol{d}(\dot{\boldsymbol{q}})$ is a damping force satisfying $\boldsymbol{d}(\dot{\boldsymbol{q}})^{T} \dot{\boldsymbol{q}}>0, \forall \dot{\boldsymbol{q}} \neq \mathbf{0}$. The most right term in (1) accounts for contact wrenches $\boldsymbol{F}_{k} \in \mathbb{R}^{6}$ acting at the contact point $k$ of the feet. Thereby, the transposed of the Jacobian matrices $\boldsymbol{J}_{k}(\boldsymbol{q})$ map the contact wrenches to the generalized forces of the bodies. A similar model has been considered in [18].

In contrast to [18], we consider compliantly actuated systems for which the joint torques $\tau \in \mathbb{R}^{n}$ are derived from the elastic potential $U(\boldsymbol{\theta}, \boldsymbol{q})$, i. e.

$$
\boldsymbol{\tau}=-\left(\frac{\partial U(\boldsymbol{\theta}, \boldsymbol{q})}{\partial \boldsymbol{q}}\right)^{T}=: \boldsymbol{\psi}(\boldsymbol{\theta}-\boldsymbol{q}) .
$$

The joint torques defined by (2) correspond to physical springs acting between the motor positions $\boldsymbol{\theta} \in \mathbb{R}^{n}$ and the joint positions $\boldsymbol{q}$. Therefore, $U(\boldsymbol{\theta}, \boldsymbol{q})$ is positive definite in the sense that $U(\boldsymbol{\theta}, \boldsymbol{q})>0, \forall(\boldsymbol{\theta}-\boldsymbol{q}) \neq \mathbf{0}$.

Remark 1: Using classical approximations [19], the motor positions are subject to dynamics $\boldsymbol{B} \ddot{\boldsymbol{\theta}}+\boldsymbol{\tau}=\boldsymbol{\tau}_{\mathrm{m}}$, where $\boldsymbol{B}$ represents the motor inertia, and the motor torque $\tau_{\mathrm{m}}$ is the control input. However, using a PD controller $\tau_{\mathrm{m}}=$ $-\boldsymbol{K}_{\mathrm{D}} \dot{\boldsymbol{\theta}}-\boldsymbol{K}_{\mathrm{P}}\left(\boldsymbol{\theta}-\boldsymbol{\theta}_{\mathrm{d}}\right)$ for the desired motor position $\boldsymbol{\theta}_{\mathrm{d}}$ with high, positive definite gain matrices $\boldsymbol{K}_{\mathrm{D}}, \boldsymbol{K}_{\mathrm{P}} \in \mathbb{R}^{n \times n}$ such that $\epsilon\left(\boldsymbol{B} \ddot{\boldsymbol{\theta}}+\boldsymbol{K}_{\mathrm{D}} \dot{\boldsymbol{\theta}}+\boldsymbol{\tau} 1\right)=\boldsymbol{\theta}_{\mathrm{d}}-\boldsymbol{\theta} \approx \mathbf{0}$, since $\epsilon:=1 /\left\|\boldsymbol{K}_{\mathrm{P}}\right\| \rightarrow 0$ (singular perturbation assumption [20]), we can approximately consider $\boldsymbol{\theta}$ as control input.

The output of the controller derived in the next section will be joint torques $\tau$, while the control input of the considered plant are motor positions $\boldsymbol{\theta}$. Since $U(\boldsymbol{\theta}, \boldsymbol{q})$ is positive definite, the functions $\boldsymbol{\psi}(\boldsymbol{\theta}-\boldsymbol{q})$ are strictly monotone. Therefore, the inverse functions $\psi(\tau)^{-1}$ exist. Using the mapping

$$
\boldsymbol{\theta}=\boldsymbol{\theta}_{0}+\Delta \boldsymbol{\theta}=\boldsymbol{\psi}(\boldsymbol{\tau})^{-1}+\boldsymbol{q},
$$

we can consider $\boldsymbol{\tau}$ and $\boldsymbol{\theta}$ as equivalent control inputs. 


\section{CONTROL APPROACH}

The goal is to control the Cartesian force acting on the center of mass (COM) of the complete floating base system via the joints. In more detail, we want to generate a periodic motion of the COM position along a predefined, one-dimensional submanifold of $\mathbb{R}^{3}$, such that the submanifold determines the "direction" of the COM motion and the resulting force. An additional goal is to change the dynamics of the plant to a minimal extent by control. This will be achieved by Cartesian impedance control [21], [22], [23], which is combined with the constraint submanifold control [17] and our recently proposed bang-bang control [15]. Compared to [21], [22], [23], and [17], where the impedance was generated partly or completely by control, we will implement the Cartesian impedance by exploiting the intrinsic, elastic behavior of the joints.

\section{A. Change of coordinates}

Consider the position of the COM $\boldsymbol{r}_{B C} \in \mathbb{R}^{3}$ with respect to a coordinate system attached to the floating base. The position $\boldsymbol{r}_{B C}=\boldsymbol{r}_{B C}(\boldsymbol{q})$ depends on the joint coordinates $\boldsymbol{q}$. In addition to $\boldsymbol{r}_{B C}(\boldsymbol{q})$, consider a virtual COM position $\boldsymbol{r}_{B C}(\boldsymbol{\theta})$ which depends on the motor position $\boldsymbol{\theta}$. In the absence of external load, the virtual position $\boldsymbol{r}_{B C}(\boldsymbol{\theta})$ reflects the equilibrium position of the spring defined by (2). Based on this consideration, we define coordinates

$$
\boldsymbol{x}(\boldsymbol{q})=\boldsymbol{r}_{B C}(\boldsymbol{q})-\boldsymbol{r}_{B C}\left(\boldsymbol{\theta}_{0}\right),
$$

where $\boldsymbol{x} \in \mathcal{X} \subset \mathbb{R}^{3}$ and $\boldsymbol{\theta}_{0}=$ const. correspond to a desired equilibrium configuration of the springs (2). These coordinates can be used to implement a Cartesian impedance between the measured COM position $\boldsymbol{r}_{B C}(\boldsymbol{q})$ and desired COM position $\boldsymbol{r}_{B C}\left(\boldsymbol{\theta}_{0}\right)$. Then, as in [17], we can consider a mapping $\boldsymbol{z}: \mathcal{X} \subset \mathbb{R}^{3} \rightarrow \mathcal{Z} \subset \mathbb{R}^{2}$ with a full rank Jacobian matrix $\boldsymbol{J}_{z}(\boldsymbol{x})$ such that

$$
\boldsymbol{z}(\boldsymbol{x})=\mathbf{0}
$$

defines a one-dimensional submanifold $\mathcal{N}_{Z}$ of $\mathbb{R}^{3}$. Once the constraint (5) is satisfied, a Cartesian impedance produces a force whose direction can be predefined by the constraint submanifold. All relevant sets and manifolds used in this approach are illustrated in Fig. 3.

\section{B. Transformation of the joint impedance}

The constraint force resulting from the intrinsic joint stiffness (2) can be derived from the elastic potential $U\left(\boldsymbol{\theta}_{0}, \boldsymbol{q}\right)=$ $U\left(\boldsymbol{\theta}_{0}, \boldsymbol{q}(\boldsymbol{x}(\boldsymbol{z}))\right)$ as follows:

$$
\begin{aligned}
\boldsymbol{\tau}_{z} & =-\left(\frac{\partial U\left(\boldsymbol{\theta}_{0}, \boldsymbol{q}(\boldsymbol{x}(\boldsymbol{z}))\right)}{\partial \boldsymbol{q}} \frac{\partial \boldsymbol{q}}{\partial \boldsymbol{x}} \frac{\partial \boldsymbol{x}}{\partial \boldsymbol{z}}\right)^{T} \\
& =-\left(\frac{\partial \boldsymbol{x}}{\partial \boldsymbol{z}}\right)^{T}\left(\frac{\partial \boldsymbol{q}}{\partial \boldsymbol{x}}\right)^{T}\left(\frac{\partial U\left(\boldsymbol{\theta}_{0}, \boldsymbol{q}\right)}{\partial \boldsymbol{q}}\right)^{T} .
\end{aligned}
$$

The last factor on the most right hand side of (6) equals the joint torques defined by (2) for $\boldsymbol{\theta}=\boldsymbol{\theta}_{0}$. From (6) it can be seen that the joint torques are transformed successively to Cartesian and constraint forces with the transposed of

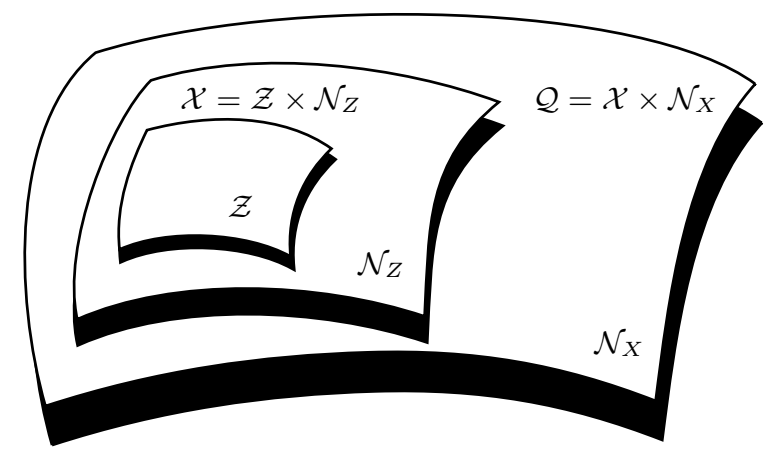

Fig. 3. The constraint $\boldsymbol{z}(\boldsymbol{x})=\mathbf{0}$ has highest priority. Together with $\mathcal{N}_{Z}$, the coordinates $\boldsymbol{x}(\boldsymbol{q})$ are completely defined in $\mathcal{X}$. The overall set $\mathcal{Q}$ is the combination of $\mathcal{X}$ and its null space $\mathcal{N}_{X}$.

the Jacobian matrices $(\partial \boldsymbol{q} / \partial \boldsymbol{x}) \in \mathbb{R}^{n \times 3}$ and $(\partial \boldsymbol{x} / \partial \boldsymbol{z}) \in$ $\mathbb{R}^{3 \times 2}$, respectively. These Jacobian matrices correspond to the Jacobian matrices of the inverse mappings of (4) and (5).

In order to derive the Jacobian matrices required in (6), we can augment the Jacobian matrices of the mappings (4) and (5) such that they are invertible. Note that thereby we avoid to define nullspace coordinates [24]. In case of (4) and (5) the procedure is as follows:

$$
\begin{gathered}
\left(\begin{array}{c}
\mathrm{d} \boldsymbol{x} \\
\mathrm{d} \boldsymbol{n}_{x}
\end{array}\right)=\boldsymbol{J}_{x}^{\mathrm{aug}} \mathrm{d} \boldsymbol{q}, \text { where } \boldsymbol{J}_{x}^{\mathrm{aug}}=\left(\begin{array}{c}
\boldsymbol{J}_{x}(\boldsymbol{q}) \\
\boldsymbol{J}_{n_{x}}(\boldsymbol{q})
\end{array}\right) \in \mathbb{R}^{n \times n}, \\
\left(\begin{array}{c}
\mathrm{d} \boldsymbol{z} \\
\mathrm{d} \boldsymbol{n}_{z}
\end{array}\right)=\boldsymbol{J}_{z}^{\mathrm{aug}} \mathrm{d} \boldsymbol{x}, \text { where } \boldsymbol{J}_{z}^{\mathrm{aug}}=\left(\begin{array}{c}
\boldsymbol{J}_{z}(\boldsymbol{x}) \\
\boldsymbol{J}_{n_{z}}(\boldsymbol{x})
\end{array}\right) \in \mathbb{R}^{3 \times 3} .
\end{gathered}
$$

The inversion of the augmented Jacobian matrices defined in (7) and (8) can be simplified with the following lemma which is proved in [25, chapt. A.5].

Lemma 1: The augmented Jacobian matrix

$$
\boldsymbol{J}^{\text {aug }}=\left(\begin{array}{c}
\boldsymbol{J} \\
\boldsymbol{J}_{n}
\end{array}\right)
$$

is a square matrix. If $\boldsymbol{J}_{n}$ is chosen as

$$
\boldsymbol{J}_{n}=\left(\boldsymbol{Z} \Theta \boldsymbol{Z}^{T}\right)^{-1} \boldsymbol{Z} \Theta
$$

where the nullspace base $Z$ satisfies $\boldsymbol{J} Z^{T}=\mathbf{0}$ and $\Theta$ is a positive definite matrix (metric), the inverse of (9) can be written in the form

$$
\left(\boldsymbol{J}^{\text {aug }}\right)^{-1}=\left(\boldsymbol{\Theta}^{-1} \boldsymbol{J}^{T}\left(\boldsymbol{J} \Theta^{-1} \boldsymbol{J}^{T}\right)^{-1} \boldsymbol{Z}^{T}\right) .
$$

Note that $\boldsymbol{Z}$ and $\Theta$ are not unique. Possible choices are discussed in the appendix.

Then, applying Lemma 1 to (7) and (8) yields

$$
\begin{aligned}
& \frac{\partial \boldsymbol{q}}{\partial \boldsymbol{x}}(\boldsymbol{q})=\boldsymbol{\Theta}_{x}^{-1} \boldsymbol{J}_{x}(\boldsymbol{q})^{T}\left(\boldsymbol{J}_{x}(\boldsymbol{q}) \boldsymbol{\Theta}_{x}^{-1} \boldsymbol{J}_{x}(\boldsymbol{q})^{T}\right)^{-1}, \\
& \frac{\partial \boldsymbol{x}}{\partial \boldsymbol{z}}(\boldsymbol{x})=\boldsymbol{\Theta}_{z}^{-1} \boldsymbol{J}_{z}(\boldsymbol{x})^{T}\left(\boldsymbol{J}_{z}(\boldsymbol{x}) \boldsymbol{\Theta}_{z}^{-1} \boldsymbol{J}_{z}(\boldsymbol{x})^{T}\right)^{-1} .
\end{aligned}
$$


From (7) and (8) it can be seen that the joint impedance (2) generates also forces in the nullspaces of $\boldsymbol{J}_{x}$ and $\boldsymbol{J}_{z}$, given by

$$
\boldsymbol{\tau}_{n_{x}}=-\boldsymbol{Z}_{x}(\boldsymbol{q})\left(\frac{\partial U\left(\boldsymbol{\theta}_{0}, \boldsymbol{q}\right)}{\partial \boldsymbol{q}}\right)^{T}
$$

and

$$
\tau_{n_{z}}=-\boldsymbol{Z}_{z}(\boldsymbol{x})\left(\frac{\partial \boldsymbol{q}}{\partial \boldsymbol{x}}(\boldsymbol{q})\right)^{T}\left(\frac{\partial U\left(\boldsymbol{\theta}_{0}, \boldsymbol{q}\right)}{\partial \boldsymbol{q}}\right)^{T},
$$

respectively. How these variables can be controlled will be suggested in the next section.

\section{Feedback control}

In the following, we introduce the control law for the Cartesian force $f_{x}$ and then present two different approaches to implement the joint torque $\tau$. The Cartesian controller comprises

$$
\boldsymbol{f}_{x}=\boldsymbol{J}_{z}(\boldsymbol{x})^{T} \boldsymbol{\tau}_{z}^{\mathrm{d}}+\boldsymbol{J}_{n_{z}}(\boldsymbol{x})^{T} \tau_{n_{z}}^{\mathrm{d}} .
$$

The first term in (16)

$$
\tau_{z}^{\mathrm{d}}=-\boldsymbol{D}_{z} \dot{z}-\boldsymbol{K}_{z} \boldsymbol{z}
$$

with symmetric and positive definite gain matrices $\boldsymbol{D}_{z} \in$ $\mathbb{R}^{2 \times 2}$ and $\boldsymbol{K}_{z} \in \mathbb{R}^{2 \times 2}$ forces the motion of $\boldsymbol{x}$ to approach the constrained submanifold defined by (5). The second term in (16)

$$
\tau_{n_{z}}^{\mathrm{d}}=\tau_{n_{z}}+\Delta \tau_{n_{z}}\left(\tau_{n_{z}}\right)
$$

is composed of a generalized force $\tau_{n_{z}}$ (15) reflecting the joint impedance on the constrained submanifold and a switching function

$$
\Delta \tau_{n_{z}}\left(\tau_{n_{z}}\right)= \begin{cases}\operatorname{sign}\left(\tau_{n_{z}}\right)\left|\hat{\tau}_{n_{z}}\right| & \text { if }\left|\tau_{n_{z}}\right|>\epsilon_{\tau_{n_{z}}} \\ 0 & \text { otherwise }\end{cases}
$$

where $\epsilon_{\tau_{n_{z}}}$ and $\hat{\tau}_{n_{z}}$ are threshold and switching constants, respectively.

In our recent papers [15] and [16] we have proposed a control similar to (19). The controller proposed in [16] excites an intrinsic mechanical oscillation mode of the plant. Compared to [16], in this work we predefine the oscillation mode by control (cf. (5) and (17)). Additionally, note that the control (19) switches the generalized force $\tau_{n_{z}}$. Thereby, we circumvent to introduce a nullspace coordinate, which is in general not possible [24].

1) Resolving the nullspace of $\boldsymbol{J}_{x}$ by preserving the initial configuration: In order to implement the joint torque $\tau$ for the Cartesian controller (16), the behavior in the nullspace of $\boldsymbol{J}_{x}(\boldsymbol{q})$ has to be specified. This can be done by projecting the intrinsic joint impedance into the nullspace of $\boldsymbol{J}_{x}(\boldsymbol{q})$ such that a nullspace force $\tau_{n_{x}}$ is generated which aims at preserving the equilibrium configuration $\boldsymbol{\theta}_{0}$. Considering the nullspace force given by (14) and the Cartesian controller (16)-(19), the joint torque $\tau$ can be implemented as

$$
\boldsymbol{\tau}=\boldsymbol{J}_{x}(\boldsymbol{q})^{T} \boldsymbol{f}_{x}+\boldsymbol{J}_{n_{x}}(\boldsymbol{q})^{T} \boldsymbol{\tau}_{n_{x}} .
$$

This has the advantage that no motor motion is required as long as $\boldsymbol{z}(\boldsymbol{x})=0$ and $\Delta \tau_{n_{z}}\left(\tau_{n_{z}}\right)=0$. Note that the controller (20) requires no knowledge of the contact states. While from a robustness point of view this might be desirable, it is not possible on the other hand to guarantee any conditions on the contact forces. This motivates us to consider the control law in IV-C.2.

2) Resolving the nullspace of $\boldsymbol{J}_{x}$ by optimal contact force distribution: In case slipping has to be avoided, the contact forces must be considered directly in the controller. To this end, an alternative approach to implement the joint torque $\tau$ for the Cartesian controller (16) is to distribute the contact forces via an optimization problem.

Therefore, consider a stacked vector of contact forces

$$
\boldsymbol{f}_{\mathrm{c}}=\left(\begin{array}{c}
\boldsymbol{f}_{1} \\
\vdots \\
\boldsymbol{f}_{n_{\mathrm{c}}}
\end{array}\right) \in \mathbb{R}^{3 n_{\mathrm{c}}},
$$

where $n_{\mathrm{c}} \in \mathbb{N}$ is the number of contact points. Additionally, consider the mapping

$$
\boldsymbol{\tau}=\boldsymbol{J}_{\mathrm{c}}\left(\boldsymbol{q}, \boldsymbol{R}_{\mathrm{b}}\right)^{T} \boldsymbol{f}_{\mathrm{c}}
$$

where the transposed of the Jacobian matrix $\boldsymbol{J}_{\mathrm{c}}\left(\boldsymbol{q}, \boldsymbol{R}_{\mathrm{b}}\right) \in$ $\mathbb{R}^{3 n_{\mathrm{c}} \times n}$ maps the contact forces $\boldsymbol{f}_{\mathrm{c}}$ to the joint torques $\boldsymbol{\tau}$. Then, we minimize the cost function

$$
E\left(\boldsymbol{f}_{c}\right)=\alpha_{1}\left\|\boldsymbol{J}_{\mathrm{c}}^{T} \boldsymbol{f}_{\mathrm{c}}-\boldsymbol{J}_{x}^{T} \boldsymbol{f}_{x}\right\|^{2}+\alpha_{2}\left\|\boldsymbol{J}_{\mathrm{c}}^{T} \boldsymbol{f}_{\mathrm{c}}-\boldsymbol{J}_{n_{x}}^{T} \boldsymbol{\tau}_{n_{x}}\right\|^{2},
$$

where the first term aims at implementing the Cartesian control (16) and the second term aims at preserving the equilibrium configuration $\boldsymbol{\theta}_{0}$, with $\alpha_{1} \gg \alpha_{2}>0$. Considering unilateral and Coulomb friction constraints for the contact forces $\boldsymbol{f}_{k}$, such that

$$
\boldsymbol{f}_{k} \in \mathcal{F}_{k}:=\left\{\boldsymbol{f}_{k} \in \mathbb{R}^{3} \mid \sqrt{f_{k_{x}}^{2}+f_{k_{y}}^{2}} \leq \mu f_{k_{z}}, f_{k_{z}} \geq 0\right\}
$$

where $\mu$ is the non-negative Coulomb friction coefficient, the optimization problem

$$
\begin{array}{ll} 
& \min E\left(\boldsymbol{f}_{c}\right) \\
\text { s.t. } & \boldsymbol{f}_{k} \in \mathcal{F}_{k}, \forall k=1, \ldots, n_{\mathrm{c}}
\end{array}
$$

can be solved ${ }^{2}$ to compute the contact forces $\boldsymbol{f}_{c}$. Note that if the unilateral / Coulomb friction constraints are removed, the control law (20) is obtained. In contrast to the approach of Sect. IV-C.1, the contact force distribution approach requires to detect the contact states.

\section{Simulation}

The proposed jumping controller has been tested in simulation for the compliantly actuated quadruped robot depicted in Fig. 4, using the articulated body algorithm [26] and a point contact version of the model [27] implemented in

\footnotetext{
${ }^{2}$ The constraint $\boldsymbol{f}_{k} \in \mathcal{F}_{k}, \forall k=1, \ldots, n_{\mathrm{c}}$ can be expressed in linear form through a polyhedral approximation of the friction cone.
} 


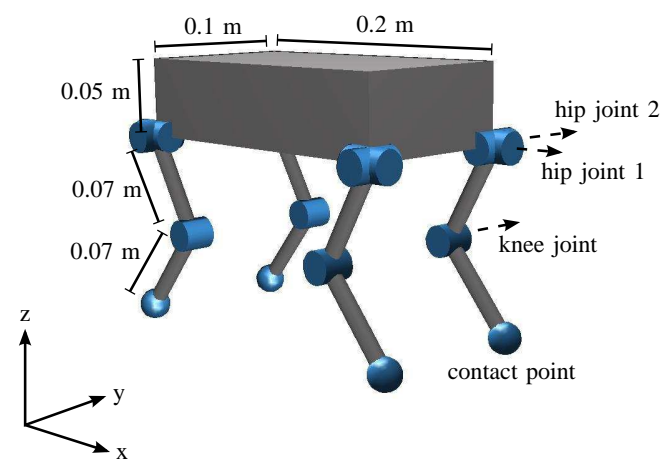

Fig. 4. Simulated quadruped: the trunk is modelled as a cuboid with mass $5 \mathrm{~kg}$ center of mass in the middle of the bottom side and inertia $\operatorname{diag}(0.0052,0.0177,0.0208) \mathrm{kgm}^{2}$. Each leg and each leg segment (thigh and shank) is identical with mass $0.1 \mathrm{~kg}$ center of mass at half the segment length and inertia (about the center of mass, perpendicular to the cylinder axis) $0.00004 \mathrm{kgm}^{2}$.

Matlab/Simulink ${ }^{\circledR}$. The considered quadruped has four legs and a total number of 12 hinge joints (two perpendicular rotation axis in each hip and one in each knee), i. e. $n=12$. The joints of each leg are actuated via linear spring $\boldsymbol{\tau}_{i}=\boldsymbol{K}_{i}\left(\boldsymbol{\theta}_{i}-\right.$ $\left.\boldsymbol{q}_{i}\right)$ with stiffness matrices $\boldsymbol{K}_{i}=\operatorname{diag}(8,4,4) \mathrm{Nm} / \mathrm{rad}$. Linear, viscous damping produces torques $\boldsymbol{d}_{i}=\boldsymbol{D}_{i} \dot{\boldsymbol{q}}_{i}$ with $\boldsymbol{D}_{i}=\operatorname{diag}(0.16,0.04,0.04) \mathrm{Nms} / \mathrm{rad}$ in the joints. Furthermore, ground contact points are considered at the tips of each leg. Thereby, a Coulomb friction constant of $\mu=0.75$ has been assumed.

\section{A. Controller implementation}

As can be seen in Fig. 4 all joint axes of the considered quadruped are parallel. Therefore, the total COM motion has been controlled in 3-dimensional space, i.e. $\boldsymbol{x}=\left(x_{\mathrm{x}}, x_{\mathrm{y}}, x_{\mathrm{z}}\right)^{T}$. To implement directed jumping motions, linear constraints of the form $z_{1}=c_{1} x_{\mathrm{x}}+c_{2} x_{\mathrm{z}}=0, z_{2}=$ $c_{3} x_{\mathrm{y}}+c_{4} x_{\mathrm{z}}=0$ have been considered. The corresponding submanifold represents a straight line passing through the origin, where $\phi=\operatorname{atan} 2\left(c_{1}, c_{2}\right)$ is the angle between the $\mathrm{x}$ axis and the line and $\psi=\operatorname{atan} 2\left(c_{3}, c_{4}\right)$ is the angle between the $y$-axis and the line.

For the nullspaces of $\boldsymbol{J}_{x}$ and $\boldsymbol{J}_{z}$ we have chosen the dynamically consistent solution given in the appendix. The proportional gain of the constraint controller (17) was chosen $\boldsymbol{K}_{z}=\operatorname{diag}(2,2) 10^{5} \mathrm{~N} / \mathrm{m}$ and the derivative gain was chosen $\boldsymbol{D}_{z}=\operatorname{diag}(1,1) 10^{4} \mathrm{Ns} / \mathrm{m}$. The parameters of the bangbang control (19) were $\epsilon_{\tau_{n_{z}}}=500 \mathrm{~N}$ and $\hat{\tau}_{n_{z}}=3500 \mathrm{~N}$. Additionally, the output of the switching function (19) has been filtered using the linear, second order filter

$$
H(s)=\frac{1}{T_{v}^{2} s^{2}+2 T_{v} s+1}
$$

where $s$ is the Laplace variable and the time constant has been chosen $T_{v}=0.01 \mathrm{sec}$.
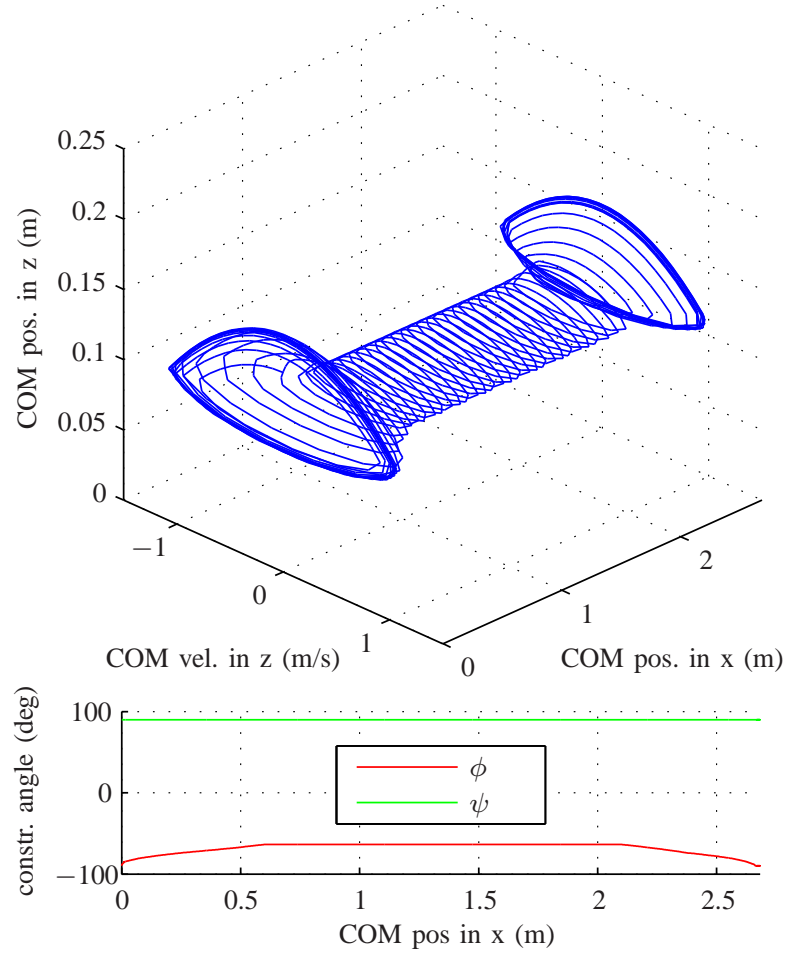

Fig. 5. Relation between the vertical velocity $\dot{r}_{\mathrm{COM}_{z}}$ and position $r_{\mathrm{COM}_{z}}$ along the horizontal position $r_{\mathrm{COM}_{x}}$ of the total COM (upper plot) and slope of the line constraint (lower plot) for a simulated sweep from vertical to forward jumping and back to vertical jumping.

\section{B. Simulation results}

It has been evaluated whether the direction of the jumping motion can be controlled using the implementation of the joint torque given in Sect. IV-C.1. Therefore, the direction of the constraint line has been varied in order to control the spatial direction of the jumping motion. Fig. 5 depicts a three-dimensional phase plot of the floating base motion and the direction of the line constraint. The phase plot shows the relation between the vertical velocity and position along the horizontal position of the total center of mass. The trunk motion of the quadruped starts with a limit cycle in the vertical direction, then evolves to a forward hopping motion and finally approaches the initially vertical limit cycle motion. Furthermore, Fig. 6 depicts the total center of mass position for 3-dimensional jumping motion. The quadruped starts with a vertical jumping motion and then evolves to a forward and sideward movement. This demonstrates the capability of the proposed method to control the direction of the jumping motion (see also the attached video).

Additionally, Fig. 7 shows the joint positions, motor positions (control input), and joint torques of one of the front leg in the vertical jumping phase. It can be observed that the joint motion is almost periodic. Furthermore, it can be seen that the motion of the motors is dominated by the filtered output of the switching control (19). This is as the submanifold is vertical and the constraint is trivially satisfied and validates one of our basic design goals changing the intrinsic mechanical behavior of the plant to a minimal extent 

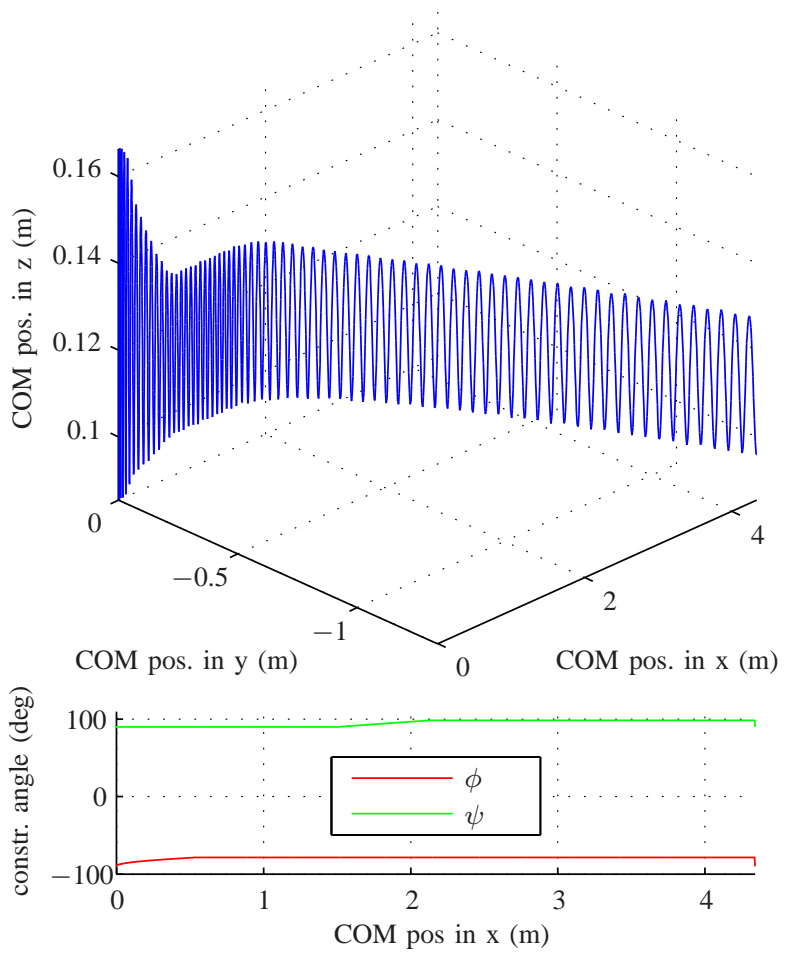

Fig. 6. Motion of the total COM (upper plot) and slope of the line constraint (lower plot) for a simulated sweep from vertical to forward and to sideward jumping.

by control.

Finally, the influence of the contact force distribution (Sect. IV-C.2) has been evaluated for a vertical jumping motion. Therefore, the lower Coulomb friction constant of $\mu=0.5$ has been assumed. To reach the limit of the friction constraint (friction cone) also the parameters of the bangbang control have been increased, i.e. $\epsilon_{\tau_{n z}}=2000 \mathrm{~N}$ and $\hat{\tau}_{n_{z}}=5500 \mathrm{~N}$. Fig. 8 compares the normal versus tangential contact force and the vertical versus horizontal movement of the tip of one leg. It can be seen that without the contact force distribution, the contact force reaches the limit of the friction cone and a horizontal movement of the tip of the leg occurs (sliding contact). This is avoided with the controller implementation of Sect. IV-C.2.

\section{CONCLUSION}

A method to control jumping motion for compliantly actuated, multilegged robots is proposed. The method applies to statically balanced legged robots, where all the joints are actuated. In particular, the controller allows to specify the desired jumping direction by defining a submanifold. Thereby, the following properties can be summarized:

- The stiffness on the submanifold is an intrinsic mechanical property of the plant;

- The force preserving the initial configuration is an intrinsic mechanical property of the plant;

- Due to the switching control the oscillation frequency adapts to the intrinsic frequency of the task;
It can be concluded that the only control actions (motor motions) are to fulfill the specified constraints and to sustain the limit cycle.

In the ideal case (where the motor is an ideal position source), it can be expected that if the specified submanifold equals the mechanical intrinsic oscillation mode of the plant, the energy required to sustain the limit cycle equals the energy dissipated in the joints and contacts. A comprehensive efficiency analysis will be part of our future work.

\section{APPENDIX}

Remark 2 (Determination of $\boldsymbol{Z}$ ): The singular value decomposition is a numerically efficient way to compute the null space base matrix $\boldsymbol{Z}$ by decomposing $\boldsymbol{J} \in \mathbb{R}^{o \times p}$ with $o<p$ such that $\boldsymbol{J}=\boldsymbol{U} \boldsymbol{S} \boldsymbol{V}^{T}$, where $\boldsymbol{U} \in \mathbb{R}^{o \times o}$ and $\boldsymbol{V} \in \mathbb{R}^{p \times p}$ are unitary matrices, and $\boldsymbol{S} \in \mathbb{R}^{o \times p}$ is a rectangular diagonal matrix containing the singular values. Herein $\boldsymbol{V}=\left(\boldsymbol{V}_{1}, \boldsymbol{V}_{2}\right)$ while $\boldsymbol{V}_{1} \in \mathbb{R}^{p \times o}$ spans the subspace of $\boldsymbol{J}$, and $\boldsymbol{V}_{2} \in \mathbb{R}^{p \times(p-o)}$ defines the null space of $\boldsymbol{J}$ so that $\boldsymbol{Z}=\boldsymbol{V}_{2}^{T}$.

Remark 3 (Choice of $\Theta$ ): In general, the metric $\Theta$ can be chosen arbitrarily, but several specific choices have beneficial properties. Two of them are briefly explained in the following. If

$$
\Theta=I
$$

then (10) simplifies to $\boldsymbol{J}_{n}=\boldsymbol{Z}$. Numerical computations can be saved that way by avoiding the inversion of $\boldsymbol{Z} \Theta \boldsymbol{Z}^{T}$ since $Z I Z^{T}=I$. Such a metric leads to a so-called static null space projection [28]. Another particular solution is

$$
\Theta=\bar{M}(q)
$$

which corresponds to the dynamically consistent formulation from the operational space approach [29] as shown in [30],
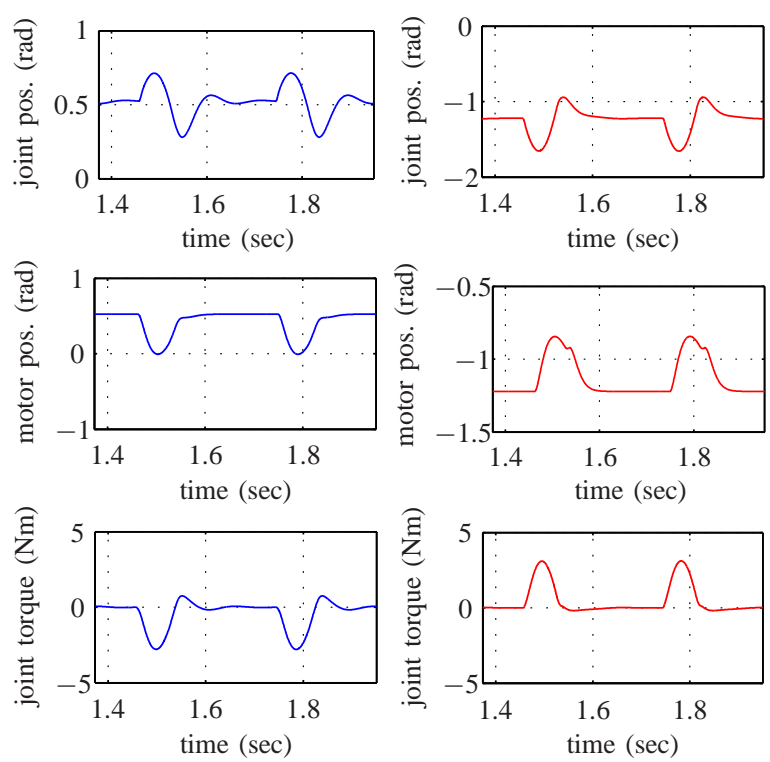

Fig. 7. Joint states corresponding to approximately two oscillation periods of the vertical jumping motion shown in Fig. 5 

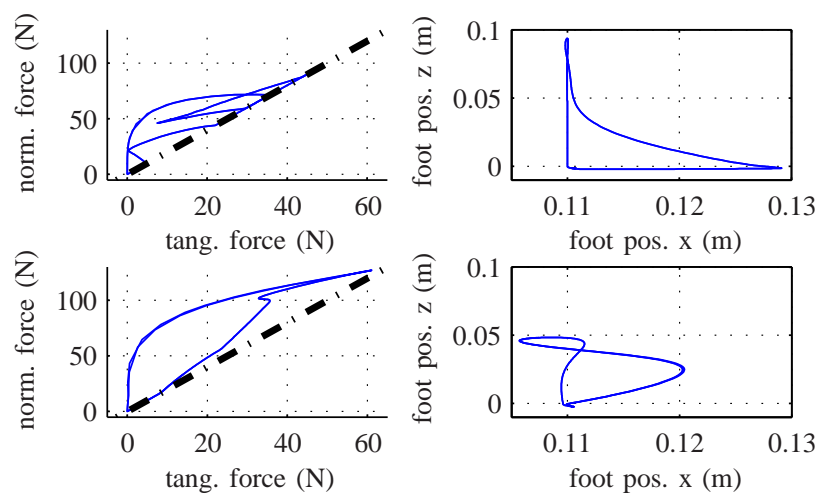

Fig. 8. Comparison of the two controller implementations: without contact force distribution cf. Sect. IV-C.1 (upper row), with contact force distribution cf. Sect. IV-C. 2 (lower row). The left column shows the tangential vs. normal contact force for one leg and the limit of the friction cone (dash-dotted line). The right column shows the motion of the tip of one leg in the xz-plane.

for example. The inertia matrix $\bar{M}(\boldsymbol{q}) \in \mathbb{R}^{n \times n}$ accounts for the complete inertia $\boldsymbol{M}(\boldsymbol{q}) \in \mathbb{R}^{(6+n) \times(6+n)}$ (including the floating base). As a consequence of this choice, the inertia matrix is of block-diagonal shape in the decoupled space ${ }^{3}$. In other words, (A2) ensures that null space actions do not lead to accelerations on the higher priority levels.

\section{REFERENCES}

[1] M. Grebenstein and P. v. d. Smagt, "Antagonism for a highly anthropomorphic hand-arm system," Advanced Robotics, vol. 22, no. 1, pp. 39-55, 2008.

[2] S. Wolf and G. Hirzinger, "A new variable stiffness design: Matching requirements of the next robot generation," in IEEE Int. Conf. on Robotics and Automation, 2008.

[3] A. Albu-Schäffer, O. Eiberger, M. Fuchs, M. Grebenstein, S. Haddadin, C. Ott, A. Stemmer, T. Wimböck, S. Wolf, C. Borst, and G. Hirzinger, "Anthropomorphic soft robotics - from torque control to variable intrinsic compliance," in Robotics Research, ser. Springer Tracts in Advanced Robotics, C. Pradalier, R. Siegwart, and G. Hirzinger, Eds. Springer, 2011, vol. 70, pp. 185-207.

[4] M. Grebenstein, A. Albu-Schäffer, T. Bahls, M. Chalon, O. Eiberger, W. Friedl, R. Gruber, S. Haddadin, U. Hagn, R. Haslinger, H. Höppner, S. Jörg, M. Nickl, A. Nothhelfer, F. Petit, J. Reill, N. Seitz, T. Wimböck, S. Wolf, T. Wüsthoff, and G. Hirzinger, "The DLR Hand Arm System," in Proc. IEEE Int. Conf. on Robotics and Automation, 2011.

[5] D. Braun, M. Howard, and S. Vijayakumar, "Exploiting variable stiffness in explosive movement tasks," in Robotics: Science and Systems, 2011.

[6] D. Braun, F. Petit, F. Huber, S. Haddadin, P. van der Smagt, A. AlbuSchaffer, and S. Vijayakumar, "Optimal torque and stiffness control in compliantly actuated robots," in Proc. IEEE/RSJ Int. Conf. on Intelligent Robots and Systems. IEEE, 2012.

[7] S. Haddadin, F. Huber, and A. Albu-Schaffer, "Optimal control for exploiting the natural dynamics of variable stiffness robots," in Proc. Int. Conf. on Robotics and Automation. IEEE, 2012, pp. 3347-3354.

[8] T. McGeer, "Passive bipedal running," Proceedings of the Royal Society of London. B. Biological Sciences, vol. 240, no. 1297, pp. 107-134, 1990.

[9] I. Poulakakis, J. A. Smith, and M. Buehler, "Modeling and experiments of untethered quadrupedal running with a bounding gait: The scout ii robot," The International Journal of Robotics Research, vol. 24, no. 4, pp. 239-256, 2005.

${ }^{3}$ The dynamic equations in the decoupled space are obtained by coordinate transformation into the local null space directions.
[10] A. Sproewitz, A. Tuleu, M. Vespignani, M. Ajallooeian, E. Badri, and A. Ijspeert, "Towards Dynamic Trot Gait Locomotion-Design, Control and Experiments with Cheetah-cub, a Compliant Quadruped Robot," International Journal of Robotics Research, vol. 32, no. 8, pp. 932 - 950, 2013.

[11] M. Hutter, C. Gehring, M. Bloesch, M. Hoepflinger, C. D. Remy, and R. Siegwart, "Starleth: A compliant quadrupedal robot for fast, efficient, and versatile locomotion," in Int. Conf. on Climbing and Walking Robots (CLAWAR), 2012.

[12] R. Full and D. Koditschek, "Templates and anchors: neuromechanical hypotheses of legged locomotion on land," J. Exp. Biol., vol. 202, no. 15 , pp. 3325-3332, 1999.

[13] A. Ijspeert, "A connectionist central pattern generator for the aquatic and terrestrial gaits of a simulated salamander," Biol. Cybern., vol. 84, pp. 331-348, 2001.

[14] J. Buchli and A. Ijspeert, "Self-organized adaptive legged locomotion in a compliant quadruped robot," Autonomous Robots, vol. 25, no. 4, pp. 331-347, 2008.

[15] D. Lakatos, F. Petit, and A. Albu-Schäffer, "Nonlinear oscillations for cyclic movements in variable impedance actuated robotic arms," in IEEE Int. Conf. on Robotics and Automation 2013, 2013.

[16] D. Lakatos, M. Görner, F. Petit, A. Dietrich, and A. Albu-Schäffer, "A modally adaptive control for multi-contact cyclic motions in compliantly actuated robotic systems," in Proc. IEEE/RSJ Int. Conf. on Intelligent Robots and Systems, 2013, pp. 5388-5395.

[17] G. Garofalo, C. Ott, and A. Albu-Schäffer, "Orbital stabilization of mechanical systems through semidefinite lyapunov functions," in Proc. American Control Conference, 2013.

[18] C. Ott, M. A. Roa, and G. Hirzinger, "Posture and balance control for biped robots based on contact force optimization," in Humanoids'11, 2011, pp. 26-33.

[19] M. Spong, "Modeling and control of elastic joint robots," Transactions of the ASME: Journal of Dynamic Systems, Measurement, and Control, vol. 109, pp. 310-319, 1987.

[20] P. Kokotovic, H. Khalil, and J. O’Reilly, Singular Perturbation Methods in Control: Analysis and Design. Academic Press, London, 1986.

[21] A. Albu-Schäffer and G. Hirzinger, "Cartesian impedance control techniques for torque controlled light-weight robots," in Proc. IEEE Int. Conf. on Robotic and Automation, 2002, pp. 657-663.

[22] C. Ott, A. Albu-Schäffer, A. Kugi, S. Stamigioli, and G. Hirzinger, "A passivity based cartesian impedance controller for flexible joint robots-part i: Torque feedback and gravity compensation," in Proc. IEEE Int. Conf. on Robotic and Automation, 2004.

[23] A. Albu-Schäffer, C. Ott, and G. Hirzinger, "A passivity based cartesian impedance controller for flexible joint robots-part ii: Full state feedback, impedance design and experiments," in Proc. IEEE Int. Conf. on Robotic and Automation, 2004.

[24] C. Ott, A. Kugi, and Y. Nakamura, "Resolving the problem of non-integrability of nullspace velocities for compliance control of redundant manipulators by using semi-definite lyapunov functions," in Proc. IEEE Int. Conf. on Robotic and Automation, 2008.

[25] C. Ott, Cartesian Impedance Control of Redundant and Flexible-Joint Robots, B. Siciliano and O. Khatib, Eds. Springer, 2008.

[26] R. Featherstone, Rigid body dynamics algorithms. Springer Berlin, 2008, vol. 49 .

[27] M. Azad and R. Featherstone, "Modeling the contact between a rolling sphere and a compliant ground plane," ACRA, Brisbane, Australia, 2010.

[28] A. Albu-Schäffer, C. Ott, U. Frese, and G. Hirzinger, "Cartesian Impedance Control of Redundant Robots: Recent Results with the DLR-Light-Weight-Arms," in Proc. IEEE Int. Conf. on Robotics and Automation, 2003, pp. 3704-3709.

[29] O. Khatib, "A Unified Approach for Motion and Force Control of Robot Manipulators: The Operational Space Formulation," IEEE Journal of Robotics and Automation, vol. RA-3, no. 1, pp. 43-53, February 1987.

[30] A. Dietrich, C. Ott, and A. Albu-Schaffer, "Multi-objective compliance control of redundant manipulators: Hierarchy, control, and stability," in Proc. IEEE/RSJ Int. Conf. on Intelligent Robots and Systems, 2013, pp. 3043-3050. 